\title{
Two-Parameter, Arbitrary Order, Exponential Approximations for Stiff Equations
}

\author{
By Byron L. Ehle and Zdenek Picel
}

\begin{abstract}
A two-parameter family of approximations to the exponential function is considered. Constraints on the parameters are determined which guarantee the approximations are $A$-acceptable. The suitability of these approximations for 2-point $A$-stable exponential fitting is established. Several numerical methods, which produce these approximations when solving $y^{\prime}=\lambda y$, are presented.
\end{abstract}

1. Introduction. Considerable attention has recently been directed toward the numerical solution of stiff differential equations. It is common practice to require that a method designed to handle such equations satisfy a condition such as

$$
\left|y_{n+1} / y_{n}\right|<1
$$

as $n \rightarrow \infty$ when solving the initial-value problem

$$
y^{\prime}=\lambda y, \quad y(0)=y_{0},
$$

using any fixed step size $h>0$ and any $\lambda$ such that $|\operatorname{Arg}(-\lambda)|<\alpha \leqslant \pi / 2$. In (1.1), $y_{n}$ is the computed solution of (1.2) at $x_{n}=n h$. Methods satisfying (1.1) with $\alpha=$ $\pi / 2$ were first considered by Dahlquist [4] and are referred to as being $A$-stable. Because many methods reduce to a computation of the form

$$
y_{k(n+1)}=E(\lambda h) y_{k n}=[E(\lambda h)]^{n+1} y_{0},
$$

for some $k \geqslant 1$ when solving (1.2), much effort has been devoted to determining if particular expressions for $E(z)$ are bounded by 1 for all $z, \operatorname{Re}(z)<0$. Approximations to the exponential satisfying this condition are said to be $A$-acceptable. It has been shown that all the diagonal and first and second subdiagonal Pade' approximations to the exponential are $A$-acceptable [5] , [15] and a number of methods have been proposed which reduce to these particular approximations [5], [10] , [13], when solving (1.2). Recently, Watts [14] has proposed several methods which are $A$-stable and reduce to the form of (1.3) when solving (1.2). Many of the exponential approximations he considers are, however, not Padé approximations. In this paper, we consider a class of functions, constructed from Padé approximations to the exponential, and depending on two parameters. Denoting the unique Padé approximation to the exponential with numerator of degree $k$ and denominator of degree $j$ by

Received February 20, 1974.

AMS (MOS) subject classifications (1970). Primary 41 A20, 30A82, 65 L05.

Key words and phrases. A-acceptable, exponential fitting, stiff equations.

Copyright $\odot$ 1975, American Mathematical Society 


$$
P_{j, k}(z)=N_{j, k}(z) / D_{j, k}(z),
$$

these approximations take the form

$$
\begin{aligned}
P_{n, 2}\left(z ; \mu_{1}, \mu_{2}\right) & =\frac{N_{n, 2}\left(z ; \mu_{1}, \mu_{2}\right)}{D_{n, 2}\left(z ; \mu_{1}, \mu_{2}\right)} \\
& =\frac{\left(1-\mu_{1}-\mu_{2}\right) N_{n, n-2}+\mu_{1} N_{n, n-1}+\mu_{2} N_{n, n}}{\left(1-\mu_{1}-\mu_{2}\right) D_{n, n-2}+\mu_{1} D_{n, n-1}+\mu_{2} D_{n, n}} .
\end{aligned}
$$

Since, for all $j, k \geqslant 0, P_{j, k}(z)-\exp (z)=O\left(z^{j+k+1}\right)$, we have at once that

$$
P_{n, 2}\left(z ; \mu_{1}, \mu_{2}\right)-\exp (z)=\left(1-\mu_{1}-\mu_{2}\right) O\left(z^{2 n-1}\right)
$$

when $n \geqslant 2$, provided $\mu_{1}+\mu_{2} \neq 1$. Thus, $P_{n, 2}\left(z ; \mu_{1}, \mu_{2}\right)$ represents a family of approximations to the exponential of arbitrarily high order. This family of approximations is the natural generalization of the families previously considered by Ehle [7] . It is easily verified that several of the exponential approximations of Watts [14] are given by appropriate choice of the parameters.

In Section 2, we consider the $A$-acceptability of such approximations. In particular, we show that if $\mu_{1} \geqslant 0, \mu_{2} \geqslant 0$ and $\mu_{1}+\mu_{2} \leqslant 1$ then $P_{n, 2}\left(z ; \mu_{1}, \mu_{2}\right)$ is $A$ acceptable. Section 3 is devoted to showing that the parameters $\mu_{1}, \mu_{2}$ may be chosen so that $P_{n, 2}\left(z ; \mu_{1}, \mu_{2}\right)$ and $e^{z}$ are equal for any two real values of $z<0$ and that all approximations of this type are also $A$-acceptable. Examples of three wellknown classes of methods which give approximations of the form (1.5) are given in Section 4. Finally, in an Appendix, we present a number of properties and results that are used in the proofs of Sections 2 and 3 of the paper. Results given in the Appendix will be denoted by A.1, A.2, etc.

2. Constraints for $A$-Acceptability of $P_{n, 2}\left(z ; \mu_{1}, \mu_{2}\right)$. To establish the $A$ acceptability of $P_{n, 2}$, we shall use the same strategy employed in [5] and [7]. Thus, we determine when $P_{n, 2}$ is bounded by 1 on the imaginary axis and also as $\operatorname{Re}(-z) \rightarrow$ $\infty$. Next, we determine when the denominator has no zeros in the left half-plane so that the Maximum Modulus Theorem may be applied to establish boundedness.

Lemma 2.1. For all $n \geqslant 2, z=i y, y$ real,

$$
\left|D_{n, 2}\left(z ; \mu_{1}, \mu_{2}\right)\right|^{2}-\left|N_{n, 2}\left(z ; \mu_{1}, \mu_{2}\right)\right|^{2} \geqslant 0 \text {, }
$$

provided

$$
\left[\left(1-\mu_{1}-\mu_{2}\right)+\frac{n-1}{2 n-1}\left(\mu_{1}+\mu_{2}\right)\right]\left[\left(1-\mu_{1}-\mu_{2}\right)+\frac{n-1}{2 n-1} \mu_{1}\right] \geqslant 0 .
$$


Proof. From the definition of $D_{n, 2}$ and $N_{n, 2}$ and the fact that $\left|N_{n, n}(i y)\right|=$ $\left|D_{n, n}(i y)\right|$ for $y$ real, we have

$$
\begin{aligned}
& \left|D_{n, 2}\right|^{2}-\left|N_{n, 2}\right|^{2} \\
& =\left(1-\mu_{1}-\mu_{2}\right)^{2}\left\{\left|D_{n, n-2}\right|^{2}-\left|N_{n, n-2}\right|^{2}\right\}+\mu_{1}^{2}\left\{\left|D_{n, n-1}\right|^{2}-\left|N_{n, n-1}\right|^{2}\right\} \\
& \quad+\left(1-\mu_{1}-\mu_{2}\right) \mu_{1}\left\{D_{n, n-2} \bar{D}_{n, n-1}-N_{n, n-2} \bar{N}_{n, n-1}\right. \\
& \left.\quad+D_{n, n-1} \bar{D}_{n, n-2}-N_{n, n-1} \bar{N}_{n, n-2}\right\} \\
& \quad+\left(1-\mu_{1}-\mu_{2}\right) \mu_{2}\left\{D_{n, n-2} \bar{D}_{n, n}-N_{n, n-2} \bar{N}_{n, n}+D_{n, n} \bar{D}_{n, n-2}-N_{n, n} \bar{N}_{n, n-2}\right\} \\
& \quad+\mu_{1} \mu_{2}\left\{D_{n, n-1} \bar{D}_{n, n}-N_{n, n-1} \bar{N}_{n, n}+D_{n, n} \bar{D}_{n, n-1}-N_{n, n} \bar{N}_{n, n-1}\right\} .
\end{aligned}
$$

The first two terms may be simplified using Lemma A.2. The third, fourth, and fifth terms can be simplified using property (A) and Lemmas A.3, A.5, and A.4, respectively, and the result follows at once.

LEMma 2.2. For all $n \geqslant 2$

$$
\underset{\operatorname{Re}(-z) \rightarrow \infty}{\operatorname{Lim}}\left|\frac{N_{n, 2}\left(z ; \mu_{1}, \mu_{2}\right)}{D_{n, 2}\left(z ; \mu_{1}, \mu_{2}\right)}\right| \leqslant 1,
$$

provided

$$
\left|\frac{\mu_{2}}{\frac{2(2 n-1)}{n-1}\left(1-\mu_{1}-\mu_{2}\right)+2 \mu_{1}+\mu_{2}}\right| \leqslant 1
$$

when $\mu_{2} \neq 0$ and for all $\mu_{1}$ otherwise.

Proof. The result follows at once from Eqs. (A.1).

Turning to the problem of establishing that $P_{n, 2}\left(z ; \mu_{1}, \mu_{2}\right)$ is analytic, we consider the region $R=\left\{\left(\mu_{1}, \mu_{2}\right) \mid \mu_{1} \geqslant 0, \mu_{2} \geqslant 0, \mu_{1}+\mu_{2} \leqslant 1\right\}$. Noting that, if $\left(\mu_{1}, \mu_{2}\right)$ satisfy the constraint of Lemma 2.1 , then the constraint of Lemma 2.2 is also satisfied, it is easily verified that $\left|P_{n, 2}\left(i y ; \mu_{1}, \mu_{2}\right)\right| \leqslant 1$ for $\left(\mu_{1}, \mu_{2}\right) \in R$ and $y$ real. We also note that, if $\mu_{1}+\mu_{2} \equiv 1$ or if $\mu_{2} \equiv 0$, then $P_{n, 2}\left(z ; \mu_{1}, \mu_{2}\right)$ reduces to the $A$-acceptable approximations studied in [7]. An attempt was made to show that $D_{n, 2}\left(z ; \mu_{1}, \mu_{2}\right)$ had no zeros in the left half-plane for $\left(\mu_{1}, \mu_{2}\right) \in R$ by observing that

$$
\begin{aligned}
D_{n, 2}\left(z ; \mu_{1}, \mu_{2}\right)= & D_{n, n-2}(z)-z\left(\frac{\mu_{1}}{2 n-1}+\frac{\mu_{2}}{n}\right) D_{n, n-2}^{\prime}(z) \\
& +\frac{z^{2} \mu_{2}}{2 n(2 n-1)} D_{n, n-2}^{\prime \prime}(z)
\end{aligned}
$$


and applying a generalization of the result of Marden employed in [7]. Using this approach, it is only possible to show that $D_{n, 2}\left(z ; \mu_{1}, \mu_{2}\right)$ has no zeros in the left halfplane when $\left(\mu_{1}, \mu_{2}\right) \in R_{n}^{*}$ where $R_{n}^{*}$ is $R$ minus a region bounded by a parabola which opens to the left and crosses the $\mu_{2}$ axis at $\mu_{2}=0$ and $\mu_{2}=2(n-1) /(2 n-1)$. Since the proof given below allows us to show that, for any $n, D_{n, 2}\left(z ; \mu_{1}, \mu_{2}\right)$ has no zeros in the left half-plane when $\left(\mu_{1}, \mu_{2}\right) \in R$, we give none of the details of the above result but mention it only for completeness.

Several preliminary results will be necessary.

LemMA 2.3. Let $(x, y)$ be any point such that $0 \leqslant x, y \leqslant 1$ and $x+y \leqslant 1$. Then there exists $\alpha, \beta$ satisfying $0 \leqslant \alpha, \beta \leqslant 1$ and such that $\alpha=y,(1-\alpha) \beta=x$.

Proof. Assume $x+y=C \leqslant 1$. Then, $y=\alpha, x=(1-\alpha) \beta$ gives $\beta=$ $(C-\alpha) /(1-\alpha) \leqslant 1$.

LEMMA 2.4. $D_{n, 2}\left(z ; \mu_{1}, \mu_{2}\right)$ can be written in the form

$$
(1-\alpha)\left[(1-\beta) D_{n, n-2}+\beta D_{n, n-1}\right]+\alpha D_{n, n},
$$

where $0 \leqslant \alpha, \beta \leqslant 1$ when $\left(\mu_{1}, \mu_{2}\right) \in R$.

Proof. Lemma 2.3.

THEOREM 2. 1. For all $n \geqslant 2, D_{n, 2}\left(z ; \mu_{1}, \mu_{2}\right)$ has no zeros in the left half-plane when $\left(\mu_{1}, \mu_{2}\right) \in R$.

Proof. In [7], it was shown, when $\mu_{2}=0$ or when $\mu_{1}+\mu_{2}=1$, that $D_{n, 2}$ has no zeros in the left half-plane. Assume that, for some $\left(\mu_{1}^{*}, \mu_{2}^{*}\right) \in R, D_{n, 2}$ has zeros in the left half-plane. Then, by the root locus property [11], there is a $\left(\mu_{1}^{*}, \mu_{2}\right) \in$ $R, \mu_{2} \neq 0$, such that $D_{n, 2}$ has a pure imaginary root, $\gamma i$. By Lemma 2.4, we may write

$$
\frac{(1-\beta) D_{n, n-2}(\gamma i)+\beta D_{n, n-1}(\gamma i)}{D_{n, n}(\gamma i)}=\frac{\alpha}{\alpha-1} \neq 0 .
$$

Now, since $\alpha /(\alpha-1)$ is real, it follows that the imaginary part on the left in (2.1) must be zero. We shall now show that this is not possible by showing that

$$
\operatorname{Im}\left(\left[(1-\beta) D_{n, n-2}+\beta D_{n, n-1}\right] \bar{D}_{n, n}\right) \neq 0
$$

for any $z=i y$. We note that (2.2) is equivalent to

$$
\frac{1}{2 i}\left\{\left[(1-\beta) D_{n, n-2}+\beta D_{n, n-1}\right] \bar{D}_{n, n}-\overline{\left[(1-\beta) D_{n, n-2}+\beta D_{n, n-1}\right]} D_{n, n}\right\}
$$

which, by property (A), can be written as

(2.3) $\frac{1}{2 i}\left\{(1-\beta)\left[D_{n, n-2} N_{n, n}-N_{n-2, n} D_{n, n}\right]+\beta\left[D_{n, n-1} N_{n, n}-N_{n-1, n} D_{n, n}\right]\right\}$. 
Now, applying Lemma A.6 and Theorem A.1, we see that, for any real $y>0$, the value of (2.3) is negative and, for any $y<0$, it is positive. Since $y=0$ is not a zero of $D_{n, 2}$, we have the contradiction which establishes that $D_{n, 2}$ has no zeros in the left half-plane for $\left(\mu_{1}, \mu_{2}\right) \in R$.

THEOREM 2.2. For all $n \geqslant 2, P_{n, 2}\left(z ; \mu_{1}, \mu_{2}\right)$ is an A-acceptable approximation to the exponential provided $\left(\mu_{1}, \mu_{2}\right) \in R$.

Proof. The result follows from Lemmas 2.1, 2.2, Theorem 2.1 and the Maximum Modulus Theorem.

We note that $R$ is not the complete region of $A$-acceptability of $P_{n, 2}$, but it is probably the region of greatest interest as will be shown by the results of the next section.

3. Exponential Approximation Using $P_{n, 2}$. The purpose of this section is to establish the following result.

THEOREM 3.1. For any $n \geqslant 2$ and any two $q_{1}, q_{2}<0$, there exists $\left(\hat{\mu}_{1}, \hat{\mu}_{2}\right) \in$ $R$, the region of A-acceptability of $P_{n, 2}$, such that

$$
P_{n, 2}\left(q_{1} ; \hat{\mu}_{1}, \hat{\mu}_{2}\right)=e^{q_{1}} \quad \text { and } \quad P_{n, 2}\left(q_{2} ; \hat{\mu}_{1}, \hat{\mu}_{2}\right)=e^{q_{2}}
$$

Proof. Observing that, with $\mu_{2}=0, P_{n, 2}$ reduces to the approximation $\widetilde{P}_{n, n-2}\left(z ; \mu_{1}\right)$ of [7] and, for $\mu_{1}=1-\mu_{2}, P_{n, 2}$ reduces to the approximation $\widetilde{P}_{n, n-1}\left(z ; \mu_{2}\right)$ of [7], we have by Theorem 5.1 of [7] that, for a given $q_{1}<0$, there exist $0 \leqslant \mu_{1}^{*}, \mu_{2}^{*} \leqslant 1$ satisfying the condition

$$
P_{n, 2}\left(q_{1} ; \mu_{1}^{*}, 0\right)=e^{q_{1}}, \quad P_{n, 2}\left(q_{1} ; 1-\mu_{2}^{*}, \mu_{2}^{*}\right)=e^{q_{1}} .
$$

Now consider any point on the line segment connecting $\left(\mu_{1}^{*}, 0\right)$ and $\left(1-\mu_{2}^{*}, \mu_{2}^{*}\right)$. Representing this line by $\mu_{2}=A^{*} \mu_{1}+B^{*}$, we have that

$$
P_{n, 2}\left(q_{1} ; \mu_{1}, A^{*} \mu_{1}+B^{*}\right)=\left(\alpha+\beta \mu_{1}\right) /\left(\gamma+\delta \mu_{1}\right)
$$

where $\alpha, \beta, \gamma$, and $\delta$ are constants determined by $q_{1}$. An expression of this form is continuous if $\gamma+\delta \mu_{1} \neq 0$ and strictly increasing, strictly decreasing, or constant. Theorem 2.1 establishes the continuity of (3.2), and then Eqs. (3.1) may be used to show that the value of (3.2) is, in fact, constant.

Now consider $P_{n, 2}\left(q_{2} ; \mu_{1}, A^{*} \mu_{1}+B^{*}\right)-e^{q_{2}}$ for $0 \neq q_{2} \neq q_{1}$. From the results of [7], we have that

$$
P_{n, 2}\left(q_{2} ; \mu_{1}^{*}, 0\right)-e^{q_{2}} \text { and } P_{n, 2}\left(q_{2} ;\left(1-\mu_{2}^{*}\right), \mu_{2}^{*}\right)-e^{q_{2}}
$$

differ in sign. Since $P_{n, 2}\left(q_{2} ; \mu_{1}, A^{*} \mu_{1}+B^{*}\right)$ is of the same form as (3.2) but with different values for $\alpha, \beta, \gamma, \delta$, and since Theorem 2.1 again establishes continuity, there is some point $\left(\hat{\mu}_{1}, \hat{\mu}_{2}\right)$ on the line segment for which $P_{n, 2}\left(q_{2} ; \hat{\mu}_{1}, \hat{\mu}_{2}\right)-e^{q_{2}}=0$. 
This completes the proof except for the special case where the line connecting the two points is vertical. It is easily seen that the same general arguments hold in that situation.

Corollary 3.1. Given $q_{1}$ and $q_{2}$, the $\hat{\mu}_{1}$ and $\hat{\mu}_{2}$ of Theorem 3.1 are unique.

Proof. This can be seen by solving for $\hat{\mu}_{1}$ and $\hat{\mu}_{2}$ in terms of $e^{q_{1}}$ and $e^{q_{2}}$, using the equalities given in Theorem 3.1.

4. Applications. The purpose of this section is to briefly illustrate several areas where exponential approximations of the form considered in this paper occur or could be employed.

As a first example, consider the method given by

$$
\sum_{i=0}^{k} \alpha_{i} h^{i} y_{n}^{(i)}+(-1)^{i+1} \beta_{i} h^{i} y_{n+1}^{(i)}=0
$$

If $\alpha_{0}=\beta_{0}=1$;

$$
\begin{gathered}
\alpha_{i}=\frac{\left(1-\mu_{1}-\mu_{2}\right)(2 k-2-i) !(k-2) !}{(2 k-2) ! i !(k-2-i) !}+\frac{\mu_{1}(2 k-1-i) !(k-1) !}{(2 k-1) ! i !(k-1-i) !} \\
+\frac{\mu_{2}(2 k-i) ! k !}{(2 k) ! i !(k-i) !}, \quad i=1,2, \ldots, k-2 ; \\
\alpha_{k-1}=\frac{\mu_{1}(k) !}{(2 k-1) !}+\frac{\mu_{2}(k-1) ! k !}{(2 k) !(k-1) !} ; \quad \alpha_{k}=\frac{\mu_{2}(k) !}{(2 k) !} \\
\beta_{i}=\frac{\left(1-\mu_{1}-\mu_{2}\right)(2 k-2-i) ! k !}{(2 k-2) ! i !(k-i) !}+\frac{\mu_{1}(2 k-1-i) ! k !}{(2 k-1) ! i !(k-i) !} \\
\quad+\frac{\mu_{2}(2 k-i) ! k !}{(2 k) ! i !(k-i) !}, \quad i=1,2, \ldots, k,
\end{gathered}
$$

it can be shown that the resulting method is, in general, of order $2 k-2$, although particular values of $\mu_{1}$ and $\mu_{2}$ produce orders of $2 k-1$ and $2 k$. For examples of these latter cases, see [7] and [6]. It is easily established that solving (1.2) wtth (4.1) using the specified coefficients gives

$$
y_{n+1}=P_{k, 2}\left(\lambda h ; \mu_{1}, \mu_{2}\right) y_{n} .
$$

From the results of Sections 2 and 3, we see that it is therefore possible to produce arbitrarily high-order $\boldsymbol{A}$-stable methods which exponentially fit at two points.

Next, consider the class of generalized Runge-Kutta methods proposed by Lawson [12] and Lawson and Ehle [13]. In these methods and also in the corresponding 
generalized Adams methods given by Jain [10], an explicit $A$ - or $L$-acceptable exponential approximation is employed. The class of approximations considered in this paper could be employed with all these methods.

As another example, consider the two-parameter implicit Runge-Kutta method given by

$$
\begin{aligned}
y_{n+1} & =y_{n}+(h / 2)\left[K_{1}+K_{2}\right], \\
K_{1} & =f\left(x_{n}+(1 / 2-\alpha) h, y_{n}+h\left[\beta_{11} K_{1}+\beta_{12} K_{2}\right]\right), \\
K_{2} & =f\left(x_{n}+(1 / 2+\alpha) h, y_{n}+h\left[\beta_{21} K_{1}+\beta_{22} K_{2}\right]\right),
\end{aligned}
$$

where

$$
\begin{aligned}
& \beta_{11}=\beta_{22}=\frac{1}{2}+\mu_{1}\left(-\frac{1}{6}\right)+\mu_{2}\left(-\frac{1}{4}\right), \\
& \beta_{12}=-\frac{1}{2}+\mu_{1}\left(\frac{2}{3}-\frac{\sqrt{3}}{6}\right)+\mu_{2}\left(\frac{3}{4}-\frac{\sqrt{3}}{6}\right), \\
& \beta_{21}=\frac{1}{2}+\mu_{1}\left(-\frac{1}{3}+\frac{\sqrt{3}}{6}\right)+\mu_{2}\left(-\frac{1}{4}+\frac{\sqrt{3}}{6}\right),
\end{aligned}
$$

and $\alpha=1 / 2+(-1 / 2+\sqrt{3} / 6)\left(\mu_{1}+\mu_{2}\right)$.

It is easily verified that this method satisfies conditions $B(2), C(1)$, and $D(1)$ of Butcher [2] and hence, by Theorem 7 of [2], the method is at least of order 2 for all $\mu_{1}$ and $\mu_{2}$. In addition, if $\mu_{1}+\mu_{2}=1, \mu_{2} \neq 1$, then $B(3)$ is true. (Actually, $B(4)$ is true, but we use only the fact that $B(3)$ is therefore true.) Employing Theorem 7 of [2], we see the method is then of order 3. Finally, if $\mu_{2}=1, \mu_{1}=0$, we obtain Butcher's unique fourth-order method.

Solving (1.2) with (4.3), it can also be verified that we obtain (4.2) with $k=2$. Based on this result and the order and stability properties of certain classes of implicit Runge-Kutta methods studied in [2], [3], [6] and [8], it is reasonable to conjecture that, for all $k \geqslant 2$, there are two-parameter implicit Runge-Kutta methods which reduce to $P_{k, 2}\left(\lambda h ; \mu_{1}, \mu_{2}\right)$ and thus could be used for double exponential fitting.

Finally, consider the 2-block one step method as proposed in Watts and Shampine [14]

$$
\left[\begin{array}{l}
y_{n+1} \\
y_{n+2}
\end{array}\right]=\left[\begin{array}{l}
1 \\
1
\end{array}\right] y_{n}
$$

$$
+h\left\{\left[\begin{array}{cc}
\left(\frac{3}{2}-2 d_{1}\right)\left(-\frac{1}{2}+d_{1}\right) \\
\left(2-2 d_{2}\right)
\end{array}\right]\left[\begin{array}{l}
f\left(y_{n+1}\right) \\
f\left(y_{n+2}\right)
\end{array}\right]+\left[\begin{array}{l}
d_{1} \\
d_{2}
\end{array}\right] \cdot f\left(y_{n}\right)\right\},
$$

$$
n=2 k, k=0,1,2, \ldots \text {, }
$$


where

$$
d_{1}=-\frac{3}{4}+\mu_{1}+\frac{7}{6} \mu_{2}, \quad d_{2}=-1+\frac{4}{3} \mu_{1}+\frac{4}{3} \mu_{2}
$$

Applying (4.4) to solve (1.2), we get, by using Cramer's rule,

$$
y_{n+2}=P_{2,2}\left(2 h \lambda ; \mu_{1}, \mu_{2}\right) y_{n} .
$$

In the special cases when $\mu_{1}=3 / 4$ and $\mu_{2}=0$, we have the second-order method described in [1], while for $\mu_{1}=0, \mu_{2}=1,(4.4)$ is the fourth-order Newton-Cotes method studied in [14]. Since $0 \leqslant \mu_{1}, \mu_{2} \leqslant 1$ and $\mu_{1}+\mu_{2} \leqslant 1$ in both cases, the corresponding methods are $A$-stable.

Appendix. In this Appendix, we summarize several of the known properties of the Padé approximations to the exponential and establish some new ones which are used to prove our main results. In particular, it is known [9] that the numerator and denominator of (1.4) satisfy

$$
N_{j, k}(z)=\sum_{m=0}^{k} \frac{(j+k-m) ! k !}{(j+k) ! m !(k-m) !} z^{m}
$$

$$
D_{j, k}(z)=\sum_{m=0}^{j} \overline{(j+k) ! m !(j-m) !}(-z)^{m}
$$

Using Eqs. (A.1), the following properties of $N_{j, k}(z)$ and $D_{j, k}(z)$, previously given in [5] and [7], are easily verified. Explicit dependence of functions on $z$ is not shown unless necessary for clarity.

(A) $\overline{N_{j, k}(i y)}=D_{k, j}(i y)$ and $\overline{D_{j, k}(i y)}=N_{k, j}(i y), y$ real.

(B) For all $j, k \geqslant 1$ and all $z$

$$
\begin{aligned}
& D_{j, k}=D_{j, k-1}+\frac{j z}{(j+k)(j+k-1)} D_{j-1, k-1}, \\
& N_{j, k}=N_{j-1, k}-\frac{k z}{(j+k)(j+k-1)} N_{j-1, k-1} .
\end{aligned}
$$

(C) For all $j, k \geqslant 1$ and all $z$

$$
N_{j-1, k}-N_{j, k-1}=\frac{z}{j+k-1} N_{j-1, k-1}, \quad D_{j-1, k}-D_{j, k-1}=\frac{z}{j+k-1} D_{j-1, k-1} .
$$

(D) For all $n \geqslant 2$ and all $z$

$$
N_{n, n}=N_{n-1, n-1}+\frac{z^{2}}{4(2 n-1)(2 n-3)} N_{n-2, n-2},
$$




$$
D_{n, n}=D_{n-1, n-1}+\frac{z^{2}}{4(2 n-1)(2 n-3)} D_{n-2, n-2} .
$$

(E) For all $j, k \geqslant 1$ and all $z$

$$
(j+k) N_{j, k}=j N_{j-1, k}+k N_{j, k-1}, \quad(j+k) D_{j, k}=j D_{j-1, k}+k D_{j, k-1} .
$$

The following less obvious results are also established in [5].

LemmA A.1. For all $n \geqslant 1$ and all $z$

$$
D_{n, n} N_{n-1, n-1}-N_{n, n} D_{n-1, n-1}=\frac{(-1)^{n} z}{(2 n-1)}\left[\frac{(n-1) ! z^{n-1}}{(2 n-2) !}\right]^{2} .
$$

LemmA A.2. For all $n \geqslant 0$ and $y$ real

$$
\left|D_{n+1, n}(i y)\right|^{2}-\left|N_{n+1, n}(i y)\right|^{2}=\left[n ! y^{n+1} /(2 n+1) !\right]^{2}
$$

and

$$
\left|D_{n+2, n}(i y)\right|^{2}-\left|N_{n+2, n}(i y)\right|^{2}=\left[n ! y^{n+2} /(2 n+2) !\right]^{2} .
$$

The following two results are found in [7].

LemmA A.3. For all $n \geqslant 2$ and all $z$

$$
\begin{gathered}
D_{n, n-2} N_{n-1, n}-N_{n, n-2} D_{n-1, n}+D_{n, n-1} N_{n-2, n}-N_{n, n-1} D_{n-2, n} \\
=(-1)^{n} \frac{2(2 n-1)}{(n-1)}\left[\frac{(n-1) ! z^{n}}{(2 n-1) !}\right]^{2} .
\end{gathered}
$$

Lemma A.4. For all $n \geqslant 1$ and all $z$

$$
\begin{gathered}
D_{n, n-1} N_{n, n}-N_{n, n-1} D_{n, n}+D_{n, n} N_{n-1, n}-N_{n, n} D_{n-1, n} \\
=(-1)^{n}\left[(n-1) ! z^{n} /(2 n-1) !\right]^{2} .
\end{gathered}
$$

Lemma A.5. For all $n \geqslant 2$ and all $z$

$$
\begin{gathered}
D_{n, n-2} N_{n, n}-N_{n, n-2} D_{n, n}+D_{n, n} N_{n-2, n}-N_{n, n} D_{n-2, n} \\
=(-1)^{n} \frac{(n-1)}{(2 n-1)}\left[\frac{(n-2) ! z^{n}}{(2 n-2) !}\right]^{2} .
\end{gathered}
$$

Proof. The result follows from property (C) and Lemma A.1.

LemmA A.6. For all $n \geqslant 2$ and all $z$ 


$$
D_{n, n-1} N_{n, n}-N_{n, n-1} D_{n, n}=\frac{-z}{2(2 n-1)}\left[D_{n, n} N_{n-1, n-1}+N_{n, n} D_{n-1, n-1}\right]
$$

and

$$
\begin{aligned}
& D_{n, n-2} N_{n, n}- N_{n-2, n} D_{n, n} \\
&=\frac{-z}{2(n-1)}\left\{\left[D_{n, n} N_{n-1, n-1}+N_{n, n} D_{n-1, n-1}\right]\right. \\
&\left.\quad+\frac{2 n(-1)^{n-1}}{2 n-1}\left[\frac{(n-1) !}{(2 n-2) !} z^{n-1}\right]^{2}\right\} .
\end{aligned}
$$

Proof. The first equality follows from property (B).

The second equality can be obtained by application of property $(E)$ and then property (B) and Lemma A.1.

LEMмA A.7. For all $n \geqslant 2$ and all $z$

$$
D_{n, n} N_{n-1, n-1}=D_{n-1, n-1} N_{n-1, n-1}+\frac{z^{2}}{4(2 n-1)(2 n-3)} D_{n-2, n-2} N_{n-1, n-1}
$$

and

$$
\begin{aligned}
D_{n, n} N_{n, n}= & D_{n, n} N_{n-1, n-1}+\frac{\dot{z}^{2}}{4(2 n-1)(2 n-3)} D_{n-1, n-1} N_{n-2, n-2} \\
& +\frac{z^{4}}{16(2 n-1)^{2}(2 n-3)^{2}} D_{n-2, n-2} N_{n-2, n-2} .
\end{aligned}
$$

Proof. Both results follow from property (D).

THEOREM A.1. For all $n \geqslant 0$ the even-powered terms of $D_{n+1, n+1}(z) N_{n, n}(z)$ and $D_{n, n}(z) N_{n, n}(z)$, denoted $E_{n+1, n}(z)$ and $E_{n, n}(z)$, respectively, are

$$
E_{n+1, n}(z)=\frac{2(n !)(n+1) !}{(2 n) !(2 n+2) !} \sum_{j=0}^{n} \frac{(2 n-2 j) !(2 n-j+1) !(-1)^{j}}{(n-j) !(n-j) ! j !} z^{2 j}
$$

$$
E_{n, n}(z)=\left[\frac{n !}{(2 n) !}\right]^{2} \sum_{j=0}^{n} \frac{(2 n-2 j) !(2 n-j) !}{(n-j) !(n-j) ! j !}(-1)^{j} z^{2 j}
$$

Proof. It is trivial to establish that $E_{n+1, n}(z)$ and $E_{n, n}(z)$ are correct for $n=$ 0 , 1. Assuming that, for some $k \geqslant 2$, the formulas given by Eqs. (A.2) for $E_{k-2, k-2}$, $E_{k-1, k-1}$, and $E_{k-1, k-2}$ are correct, it is possible to show that the formulas for $E_{k, k}$ and $E_{k, k-1}$ as given by Eqs. (A.2) are also correct. This is done by observing that Lemma A.7 implies that 


$$
\begin{aligned}
E_{k, k-1} & =E_{k-1, k-1}+\frac{z^{2}}{4(2 k-1)(2 k-3)} E_{k-1, k-2}, \\
E_{k, k} & =E_{k, k-1}+\frac{z^{2}}{4(2 k-1)(2 k-3)} E_{k-1, k-2}+\frac{z^{4}}{16(2 k-1)^{2}(2 k-3)^{2}} E_{k-2, k-2},
\end{aligned}
$$

since the even-powered terms of $D_{n-2, n-2} N_{n-1, n-1}$ and $D_{n-1, n-1} N_{n-2, n-2}$ are the same. Thus, the proof is completed by induction.

Acknowledgement. The results given above were obtained while both authors were in the Department of Electrical and Computer Engineering of Syracuse University. Support by both the National Research Council of Canada (Grant \#A7637) and the University of Victoria was also received during the preparation of this paper.

Department of Mathematics

University of Victoria

Victoria, British Columbia, Canada

1. T. A. BICKART \& Z. PICEL, "High order stiffly stable composite multistep methods for numerical integration of stiff differential equations," (BIT), v. 13, 1973, pp. 272-286.

2. J. C. BUTCHER, "Implicit Runge-Kutta processes," Math. Comp., v. 18, 1964, pp. 50-64. MR 28 \#2641.

3. F. H. CHIPMAN, “ $A$-stable Runge-Kutta processes,” (BIT), v. 11, 1971, pp. 384-388. MR 45 \#4648.

4. G. G. DAHLQUIST, "A special stability problem for linear multistep methods," (BIT), v. 3, 1963, pp. 27-43. MR 30 \#15.

5. B. L. EHLE, " $A$-stable methods and Padé approximation to the exponential," SIAM J. Math. Anal., v. 4, 1973, pp. 671-680.

6. B. L. EHLE, "High order $A$-stable methods for the numerical solution of systems of D. E's," (BIT), v. 8, 1968, pp. 276-278. MR $39 \# 1119$.

7. B. L. EHLE, Some Results on Exponential Approximation and Stiff Equations, Univ. of Victoria Res. Rep. \#77, Victoria, B. C., Canada, January 1974.

8. B. L. EHLE, On Padé Approximations to the Exponential Function and A-Stable Methods for the Numerical Solution of Initial Value Problems, Res. Rep. CSRR2010, Dept. of Appl. Anal. and Comput. Sci., University of Waterloo, Canada.

9. P. M. HUMMEL \& C. L. SEEBECK, "A generalization of Taylor's theorem," Amer. Math. Monthly, v. 56, 1949, pp. 243-247. MR 10, 516.

10. R. K. JAIN, "Some $A$-stable methods for stiff ordinary differential equations," Math. Comp., v. 26, 1972, pp. 71-77. MR 46 \#2869.

11. ALLAN M. KRALL, "The root locus method: A survey," SIAM Rev., v. 12, 1970, pp. 64-72. MR $41 \# 5078$.

12. J. D. LAWSON, "Generalized Runge-Kutta processes for stable systems with large Lipschitz constants," SIAM J. Numer. Anal., v. 4, 1967, pp. 372-380. MR 36 \#4811.

13. J. D. LAWSON \& B. L. EHLE, Improved Generalized Runge-Kutta, Proc. Canadian Computer Conference, Montreal, June 1972.

14. H. A. WATTS \& L. F. SHAMPINE, " $A$-stable block implicit one-step methods," $(B I T)$, v. 12, 1972, pp. 252-266. MR $46 \# 6603$.

15. R. S. VARGA, "On higher order stable implicit methods for solving parabolic partial differential equations," J. Math. and Phys., v. 40, 1961, pp. 220-231. MR 25 \#3613. 